\title{
INTER-ISLAND ENERGY TRANSFER IN AlGaAs/GaAs QUANTUM WELLS GROWN BY MOLECULAR BEAM EPITAXY
}

\author{
M. GodLEWSKI \\ Institute of Physics, Polish Academy of Sciences \\ Al. Lotników 32/46, 02-668 Warsaw, Poland \\ P.O. Holz, J.P. Bergman, B. Monemar
}

Dept. of Physics and Meas. Technol., Linköping Univ., 58183 Linköping, Sweden

K. REgiŃSKI AND M. BUgajSKI

Institute of Electron Technology, Al. Lotników 32/46, 02-668 Warsaw, Poland

The results of photoluminescence, time-resolved photoluminescence, photoluminescence excitation and photoluminescence kinetics studies are presented for a $\mathrm{Al}_{0.3} \mathrm{Ga}_{0.7} \mathrm{As} / \mathrm{GaAs}$ quantum well system grown without growth interruptions at the interfaces. The time-resolved photoluminescence measurements show drift of excitons towards lower energy states induced in a quantum well by potential fluctuations. We present also a first direct evidence for migration of free excitons from the 24 to $25 \mathrm{ML}$ regions of the quantum well and interpret these results within a linear rate model, deriving the transition rate of $290 \mathrm{ps}^{-1}$. Such inter-island migration processes have been observed till now only in growth interrupted structures.

PACS numbers: 78.66.Fd, 78.47.+p, 73.20.Jc

\section{Introduction}

Exciton migration between different islands in a quantum well (QW) of varying thickness has been observed experimentally for growth interrupted GaAs/AlGaAs QW structures (see e.g. Refs. [1-3]). Such exciton migration is a consequence of fluctuations in a $\mathrm{QW}$ width of a planar size larger, much larger than exciton radius. Potential fluctuations in a QW $[4,5]$ can also give rise to exciton localization [6] and Stokes shift between the heavy hole exciton lines observed in photoluminescence (PL) and PL excitation (PLE) studies [7].

In this communication we present the results of PL, PLE and PL kinetics and time-resolved PL measurements explicitly demonstrating the complex nature of excitons in a high quality GaAs/AlGaAs QW structure grown without growth 
interruptions at interfaces. By selectively exciting the PL emission (SPL spectrum) we resolve several PL components and relate them to the presence of large (compared to the exciton radius), relatively flat regions of the $\mathrm{QW}$ differing in width by one monolayer. This effect was observed previously only for samples grown with growth interruptions at the interfaces. The in-plane and inter-island migration of excitons is demonstrated and its time scale is determined.

\section{Experimental}

The PL experiments were performed on an $\mathrm{Al}_{0.3} \mathrm{Ga}_{0.7} \mathrm{As} / \mathrm{GaAs} \mathrm{QW}$ structure grown at $630^{\circ} \mathrm{C}$ on the (001) oriented semi-insulating GaAs substrate covered with $1 \mu \mathrm{m}$ thick undoped GaAs epilayer by molecular beam epitaxy using a Riber $32 \mathrm{P}$ system. The structure was grown without growth interruptions at the interfaces. The PL study was performed for nominally 25 monolayer thick QW confined between $400 \AA$ thick AlGaAs barriers. The structure was capped with a $100 \AA$ thick undoped GaAs layer. The PL, PLE and PL kinetics measurements were performed at $2 \mathrm{~K}$ on experimental setups described elsewhere [8].

\section{Results and discussion}

In Fig. $1(a)$ we show PL spectrum observed under a nonresonant excitation, where the excitation energy falls into the continuum of electron and hole states, but still lies below the AlGaAs barrier energy. The spectrum measured for a resonant excitation condition (resonant excitation when a photon energy coincides with one of the heavy hole (HH) or light hole (LH) exciton energies) is shown in Fig. $1(b)$. Distinct differences between the two PL spectra are visible. We decomposed the resonant excitation PL spectrum into three Gaussian components, centered at $1.5852 \mathrm{eV}, 1.5836 \mathrm{eV}$ and $1.5815 \mathrm{eV}$. Based on the results of theoretical calculations and the PLE experiments shown below, we attribute these PL components to the free exciton (FE) recombination in the 24 monolayer wide (FE $24 \mathrm{ML}$ ) (1.5852 eV PL) and 25 monolayer wide (FE 25 ML) (1.5815 eV PL) regions in the QW plane. The $1.5836 \mathrm{eV}$ PL is assigned to the neutral donor bound exciton (DBE) recombination in the $24 \mathrm{ML}$ wide region of the QW (DBE $24 \mathrm{ML}$ ). The full width at half maximum of these components is $2.1 \mathrm{meV}, 1.5 \mathrm{meV}$ and $2.3 \mathrm{meV}$ for the FE $24 \mathrm{ML}$, DBE $24 \mathrm{ML}$ and FE $25 \mathrm{ML}$ emissions, respectively. The PL spectrum measured under the nonresonant excitation conditions is dominated by a single line, which we associate with the DBE 25 ML PL. The FE 24 ML, FE 25 ML and DBE $24 \mathrm{ML}$ PL emissions observed under the resonant excitation contribute to a weak high energy wing of the observed PL band. We note also the presence of a partly resolved low energy component of the PL emission with a maximum at about $1.579 \mathrm{eV}$. This PL is much broader than the other four and is thus likely of different origin, as will be discussed later on.

The assignment of the FE $24 \mathrm{ML}$ and FE $25 \mathrm{ML}$ PL emissions is based on the PLE results presented in Fig. 2 and theoretical modeling results discussed elsewhere [8]. The PLE spectra were measured with the detection set at different photon energies within the PL spectrum. Figure $2(a)$ shows the PLE spectrum measured for the detection set at the FE $24 \mathrm{ML}$ emission (1.5854 eV PL) and in Fig. $2(b)$ the detection was set at the DBE $25 \mathrm{ML}$ emission (1.5800 eV PL). The 


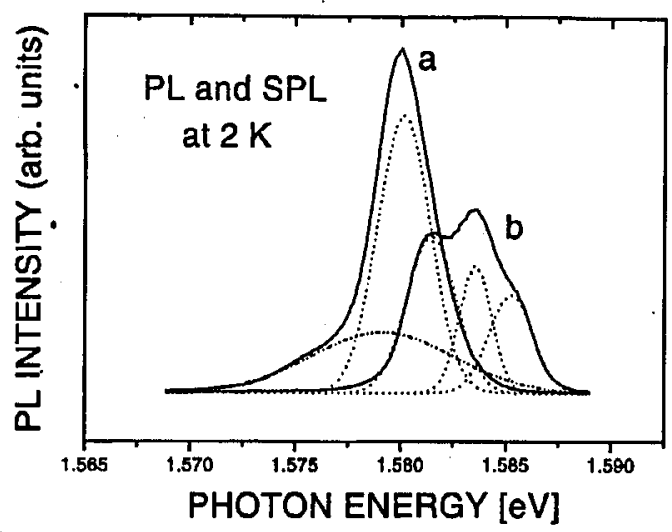

Fig. 1. $\quad a$ - the photoluminescence spectrum measured at $2 \mathrm{~K}$ for a nonresonant excitation at $1.6978 \mathrm{eV}$. Curve $b$ shows the SPL spectrum measured for a resonant excitation into the HH exciton at $1.5869 \mathrm{eV}$ (HIH FE $24 \mathrm{ML}$ ). Similar PL was observed for the excitation into the LH FE $24 \mathrm{ML}(1.6067 \mathrm{eV})$. The dotted lines show contributions from the recombination of FE $24 \mathrm{ML}, \mathrm{DBE} 24 \mathrm{ML}, \mathrm{FE} 25 \mathrm{ML}$ and DBE $25 \mathrm{ML}$ excitons and of a broad PL band, tentatively identified with a free-to-bound transition.

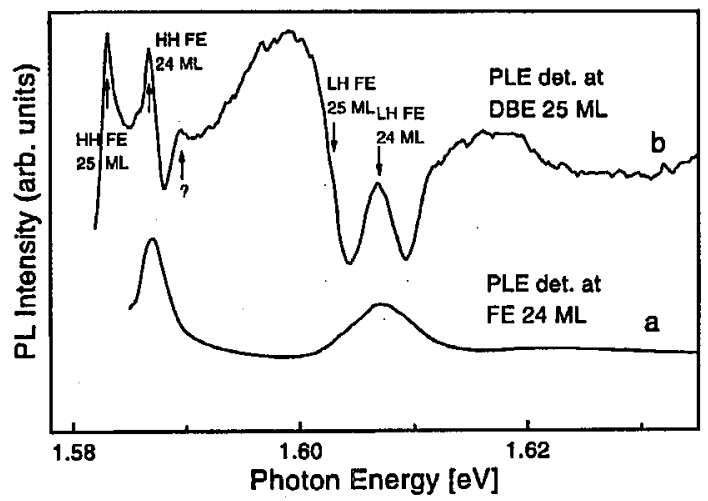

Fig. 2. Photoluminescence excitation spectrum for detection energy set at the peak of the FE $24 \mathrm{ML}$ emission (1.5852 eV) (a) and at the peak of the DBE $25 \mathrm{ML}$ emission $(1.5802 \mathrm{eV})(b)$.

PLE spectra measured with the detection set at other photon energies contained similar information. We attribute the sharp PLE peak at $1.5869 \mathrm{eV}$ (Fig. $2(a)$ ) to the heavy-hole free exciton peak of the FE $24 \mathrm{ML}$ emission. The LH exciton peak is observed at the higher energy.

The PLE curve detected at the DBE 25 ML peak (Fig. $2(b)$ ) shows a number of additional features compared to the one in Fig. $2(a)$. It contains an additional heavy hole exciton peak at $1.5831 \mathrm{eV}$ (HH FE $25 \mathrm{ML}$ ) and two broad bands with the maxima at about $1.599 \mathrm{eV}$ and $1.618 \mathrm{eV}$. The first of them overlaps with the $\mathrm{LH}$ 
FE 25 ML peak, which thus could not be resolved in our study. We explain these two broad PLE bands, with maxima at $1.599 \mathrm{eV}$ and at $1.618 \mathrm{eV}$, by transitions to continuum states of the first confined electron/heavy hole and electron/light hole pairs. By shifting the detection energy we observed that the appearance of these two broad PLE peaks correlates with the observation of a PL spectrum peaked at $1.579 \mathrm{eV}$. We tentatively assign this PL band to the free-to-bound (free electron-acceptor) optical transition.

We underline the presence of the HH and LH FE 24 ML peaks in the PLE (see Fig. $2(b)$ ) detected at DBE 25 ML PL. We explain this fact by excitation transfer from the FE $24 \mathrm{ML}$ to the FE $25 \mathrm{ML}$ and then DBE $25 \mathrm{ML}$. The temporal evolution of this transfer was studied in detail. To quantify the effects of PL time decay and exciton migration, we decomposed the time-resolved PL spectra into three (resonant excitation) and twn (nonresonant excitation) Gaussian components and traced their time and intensity evolution for the FE $24 \mathrm{ML}, \mathrm{DBE} 24 \mathrm{ML}, \mathrm{FE}$ $25 \mathrm{ML}$ and DBE $25 \mathrm{ML}$ PL emissions. We observed that the DBE $24 \mathrm{ML}, \mathrm{FE}$ $25 \mathrm{ML}$ and DBE $25 \mathrm{ML}$ PLs move towards lower energies during excitons decay, with the rate of about $0.97 \mathrm{meV} / \mathrm{ns}$ (DBE $24 \mathrm{ML}$ ), $1.34 \mathrm{meV} / \mathrm{ns}$ (FE $25 \mathrm{ML}$ ) and $1.03 \mathrm{meV} / \mathrm{ns}$ for the DBE $25 \mathrm{ML}$ PL. Such motion of exciton lines towards lower energies reflexes their drift-diffusion [5].

The PL decay times of individual PL subbands were determined from the deconvolution of the time-resolved PL spectra. To evaluate the rate of excitons migration rate we notice a clear difference in the PL decay times for the two FE emissions. The decay time of the FE $24 \mathrm{ML}$ emission is $115 \mathrm{ps}$, while the FE $25 \mathrm{ML}$ decays with the time constant of $190 \mathrm{ps}$. The $75 \mathrm{ps}$ difference between the decay times of the two FE emissions is too large to be explained by a difference in the radiative decay time of two QWs differing in width by only one monolayer [6]. We relate this difference to the effect of exciton migration from $Q W$ regions 24 monolayers wide to regions 25 monolayers wide. From the linear rate equations we derive the exciton transfer rate from the 24 to the $25 \mathrm{ML}$ region to be approximately $290 \mathrm{ps}^{-1}$. Similar transfer rate was given by Deveaud et al. [3] for $\mathrm{GaAs} / \mathrm{AlGaAs}$ structure grown with growth interrupts at the interfaces. Finally, we point out that the measured Stokes shifts of $1.5-2.0 \mathrm{meV}$ and the observed FE line width of about $2 \mathrm{meV}$ correlate well with the theory reported by Fang Yang et al. [7].

Concluding, we present the direct evidence of the inter-islands (large regions of flat interfaces) exciton migration for the GaAs/AlGaAs MBE structure grown without growth interruptions at interfaces.

This work was partly supported by grant 8T 11B 05608 of the Committee for Scientific Research.

\section{References}

[1] M.A. Herman, D. Bimberg, J. Christen, J. Appl. Phys. 70, R1 (1991).

[2] M. Kohl, D. Heitmann, S. Tanaka, K. Leo, K. Ploog, Phys. Rev. B 39, 7736 (1989).

[3] B. Deveaud, T.C. Damen, J. Shah, C.W. Tu, Appl. Phys. Lett. 51, 828 (1987).

[4] C. Weisbuch, R. Dingle, A.C. Gossardand, W. Wiegmann, Solid State Commun. 38, 709 (1981). 
[5] M. Zachau, J.A. Kash, W.T. Masselink, Phys. Rev. B 44, 8403 (1991).

[6] M. Bugajski, M. Godlewski, J.P. Bergman, B. Monemar, K. Regiński, M. Kaniewska, Thin Solid Films 267, 84 (1995).

[7] F. Yang, M. Wilkinson, E.J. Hustin, K.B. O'Dowell, Phys. Rev. Lett. 70, 323 (1993).

[8] M. Godlewski, P.O. Holz, J.P. Bergman, B. Monemar, K. Regiński, M. Bugajski, E.M. Goldys, T.L. Tansley, to be published. 